Laryng. Rhinol. Otol. 62 (1983) 320-327

(c) 1983 Georg Thieme Verlag Stuttgart - New York

\section{Poröse Kunststoffe für die Ohrmuschelplastik}

\author{
A. Berghaus, M. Axhausen, M. Handrock*
}

Klinikum Steglitz der Freien Universität Berlin, Hals-Nasen-Ohrenklinik und Poliklinik (Leiter: Prof. Dr. D. Zühlke)

Zur Rekonstruktion der Ohrmuschel bedarf es neben ausreichend geeigneter Haut auch eines stabilen Stützgerüstes. Von diesem Gerüst wird neben hoher Formbeständigkeit, guter Gewebeverträglichkeit und geringer Resorptionsquote auch die Fähigkeit zur Reliefbildung durch gute Adaptation der Haut erwartet. Am weitesten verbreitet ist hier wohl die Verwendung von autogenem Knorpel aus der Rippe (Tanzer (15), Brent (4), Fukuda (7), Meyer (11), Weerda (16)) oder seltener aus der Ohrmuschel der anderen, nicht betroffenen Seite (Gorney (8)). Peer (13) hat Knorpelsplitter aus der Rippe des Patienten zunächst in einer Metallform aus Vitallium unter der Bauchhaut zu einem geformten Gerüst zusammenwachsen lassen. Jedoch gibt es zahlreiche Berichte über die Resorption nicht nur von allogenem, sondern auch autogenem Knorpel (Matthews (9), Spina (14)). Dies gilt besonders für kleine und kleinste Knorpelsplitter (Breadon (3)). So hatten auch unsere eigenen Versuche zur Verwendbarkeit von fibrinvernetzten Knorpelspänen für die Rekonstruktion der Ohrmuschel gezeigt, daß nicht nur xenogene, sondern auch allound sogar autogene Späne, die zunächst durch Vernetzen mit Fibrinkleber ein stabiles Gerüst abgaben, wegen beschleunigter Resorption zumindest im Tierversuch keinen Erfolg versprachen (1).

Wegen ihrer leichten Formbarkeit, der meist guten Formstabilität, der schnellen, sterilen Verfügbarkeit und der gegenüber Knorpel geringeren Resorptionsgefahr sind auch zahlreiche Kunststoffe zum Einsatz gekommen, wie etwa Acryl (Matthews (9)), Nylon (Meyer (10), Silikon (Obmori (12)), um nur einige zu nennen (vergl. auch Cronin (6)).

Die vielfach Kunststoffen gegenüber bestehenden Vorbehalte wegen des Risikos von Infektion, Hautnekrose und Implantatabstoßung konnten eigene Untersuchungen in Übereinstimmung mit den Ergebnissen von Brown (5) nicht allgemein bestätigen (1). Zwar war bei unseren ersten Versuchen langzeitig die Formstabilität wegen der dabei gewählten Versuchsanordnung mit Zerkleinerung des Kunststoffes nicht ausreichend, jedoch war die Gewebeverträglichkeit des verwendeten porösen Polyäthylens ausgezeichnet.

Dieses positive Ergebnis und die Aussicht, auf die Rippenknorpelentnahme für die Rekonstruktion der Ohrmuschel verzichten zu können, veranlaßten uns, 4 moderne, poröse Kunststoffe in einer tierexperimentellen Untersuchung auf ihre Eignung für die Ohrmuschelplastik zu untersuchen.

\footnotetext{
* Auszugsweise vorgetragen auf der 53. Jahresversammlung der Deutschen Gesellschaft für Hals-Nasen-Ohren-Heilkunde, Kopf- und Halschirurgie in Bad Reichenhall, Mai 1982.
}

\section{Zusammenfassung}

Vier poröse Kunststoffe - Dacron, Poröses Polyäthylen, Proplast II und Teflon - wurden auf ihre Eignung als Gerüst für die Ohrmuschelrekonstruktion untersucht. Dazu wurden bis zu $1,8 \mathrm{~mm}$ starke, gefaltete Implantate mit verschiedenen Techniken unter die Bauchhaut von Ratten verpflanzt, um dort eine stehende Hautfalte zu bilden.

Die Verwendung von Fibrinkleber erwies sich dabei als nützlich. Von den Kunststoffen überzeugte nach 10 Monaten in erster Linie das Poröse Polyäthylen, welches in der Handhabung den anderen Materialien gegenüber Vorteile bietet und dauerhaft seine Form behält. Dieser Kunststoff kam auch bei der Ohrmuschelrekonstruktion in der Klinik zur Anwendung.

\section{Porous Synthetic Materials in External Ear Reconstruc-} tion

An investigation was done on the suitability of four porous synthetic materials - Dacron, porous polyethylene, Proplast II and Teflon - for use as a framework in external ear reconstruction. For this purpose, folded implants up to $1,8 \mathrm{~mm}$ thick were implanted under the abdominal skin of rats to create a standing skin fold. The fibrin glue proved useful. After 10 months the most convincing of the synthetic materials was porous polyethylene, which offered advantages over the other materials in handling, and retained a lasting form.

This synthetic was also used in clinical reconstruction of the external ear.

\section{Material und Methode}

Für die Untersuchung ausgewählt wurden folgende Kunststoffe: 1. Poröses Polyäthylen. Stärke: ca. 1,2 mm, Porengröße: 40-200 fu. Porosität: $40 \%$ (Abb. 1).

2. Teflonfilz. Stärke: $1,8 \mathrm{~mm}$. Porengröße nach eigenen rasterelektronenmikroskopischen Messungen: 50-200/u (keine Herstellerangaben) (Abb. 2).

3. Sauvage filamentöses Dacron-Velour-Patch. Stärke: 0,6 mm. Mittlere Porosität $1600 \mathrm{ccm}$ (Abb. 3).

4. Proplast II Sheeting. Stärke $1 \mathrm{~mm}$. Bestehend aus Teflon-Fluorocarbonpolymer und Aluminiumfaser. Im Gegensatz zum schwarzen Proplast I, bei dem Carbonfaser verwendet wurde, ist Proplast II weiß. Porosität $70-90 \%$. Porengröße $100-400$ u (Abb. 4).

Alle untersuchten Kunststoffe besitzen offene Poren. Die Versuchstiere waren $200 \mathrm{~g}$ schwere, weiße Ratten. Der Versuchsaufbau sollte modellhaft den klinischen Erfordernissen Rechnung tragen. Deshalb wurden die $1 \times 2,5 \mathrm{~cm}$ großen Implantate mit einer Falte bzw. einem Relief versehen. Bei Teflon, Dacron und Proplast II wurde die Faltung durch eine feine Suturamidnaht erreicht (Abb. 5, 6, 7); das thermoplastische Polyäthylen dagegen wurde unter Erwärmen in eine Form gebracht, die dem Querschnitt einer Ohrmuschel etwa entsprach (Abb. 8).

Diese Implantate wurden dann mit Fibrinkleber* benetzt und schließlich nach der seit 1980 von uns benutzten Methode (1) in subkutane Taschen unter der Bauchhaut der Ratten implantiert (Abb. 9). Dort geschah die Fixierung in der gewünschten Position im Hauptteil der

\footnotetext{
* Tissucol \pm Immuno GmbH., Heidelberg.
} 


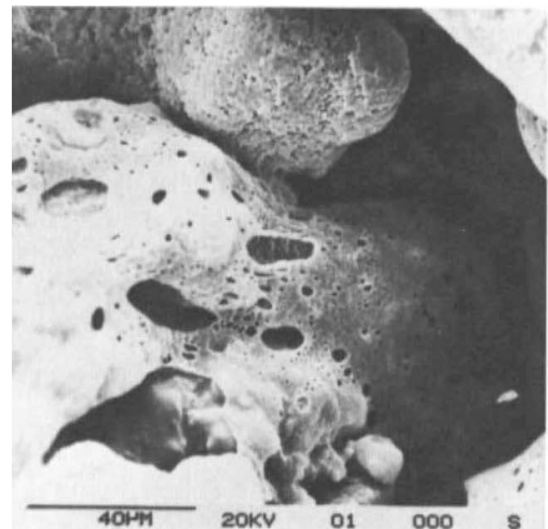

Abb. 1 Poröses Polyäthylen. Rasterelektronenmikroskop; Vergrößerung ca. 500 fach.

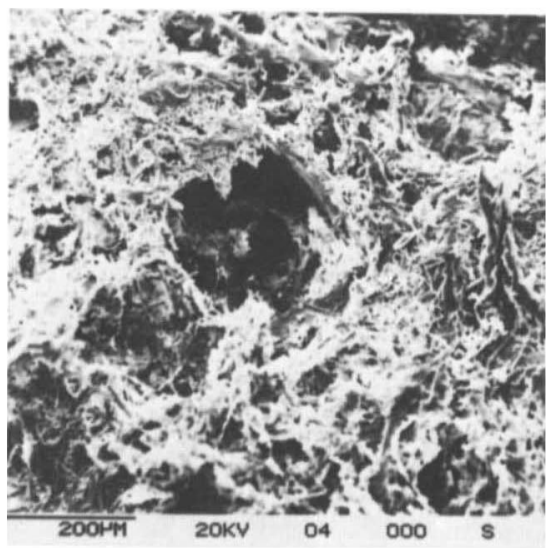

Abb. 4 Proplast II. Rasterelektronenmikroskop; Vergrößerung ca. 100fach.

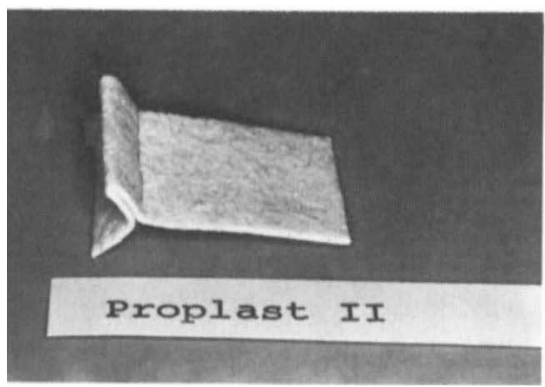

Abb. 7

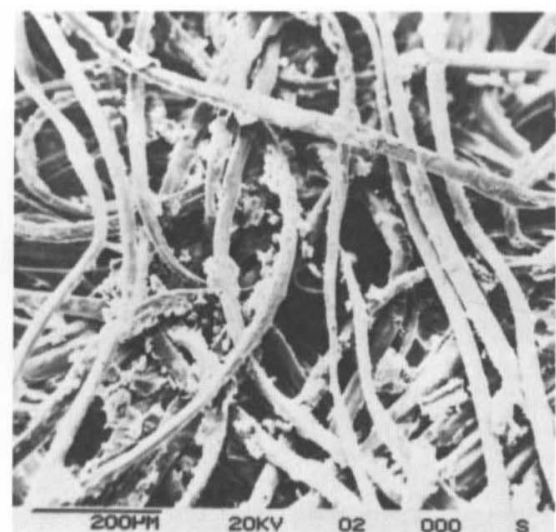

Abb. 2 Tefion felt. Rasterelektronenmikroskop; Vergrößerung ca. 100 fach.

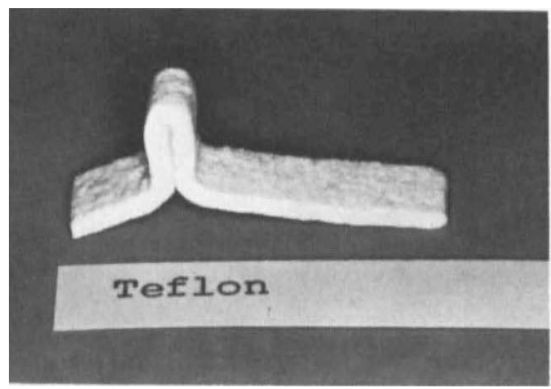

Abb. 5 Gefaltetes Teflon-Implantat vor Einpflanzung.

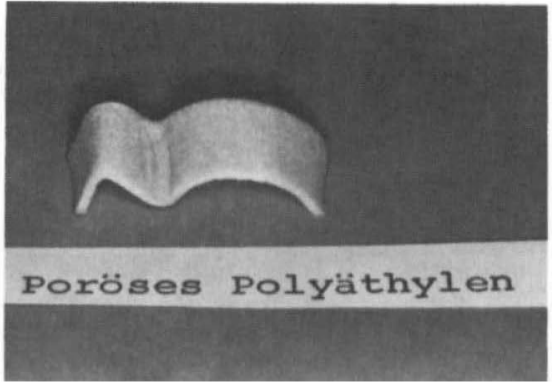

Abb. 8

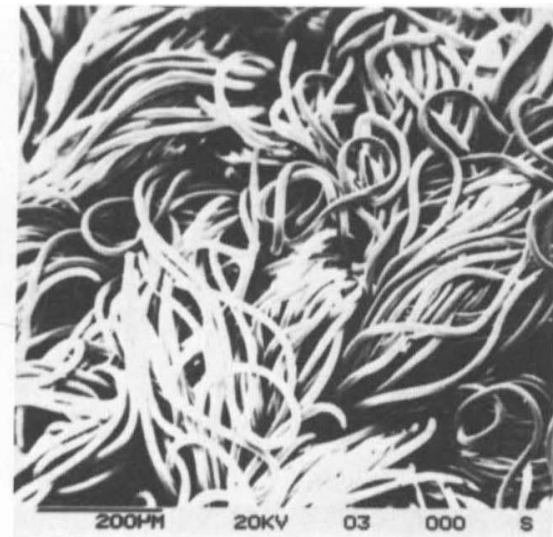

Abb. 3 Dacron Velour. Rasterelektronenmikroskop; Vergrößerung ca. 100fach.

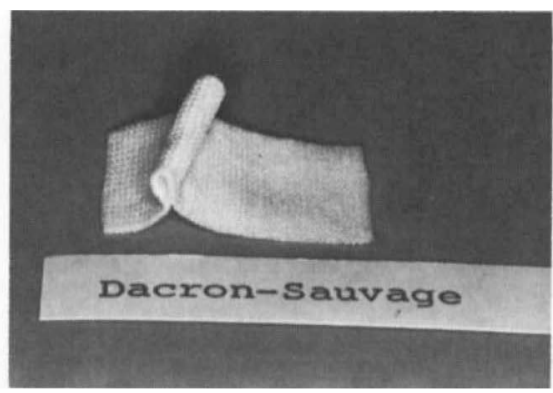

Abb. 6 Dacron-Implantat

Abb. 7 Proplast-Implantat.

Abb. 8 Unter Erhitzen auf ca. $150^{\circ} \mathrm{C}$ in Form gebrachtes Polyäthylen-Implantat.
Versuche wiederum durch Fibrinkleber (Abb. 10), zusätzlich durch eine die Hautbedeckung sowie den Kunststoff greifende Matratzennaht (Ább. 11).

50 Implantate wurden nach maximal 10 Monaten rasterelektronenmikroskopisch und histologisch nachuntersucht.

\section{Ergebnisse}

Zum Vergleich mit der Fibrinfixierung durchgeführte Implantationen ohne Fibrinkleber zeigten, daß das Einsetzen der Implantate in die gewünschte Position und die
Adaption der Haut dann erheblich erschwert sind. Eine Auswirkung des Klebers auf Art oder Geschwindigkeit des Einsprossens von Bindegewebe in die porösen Kunststoffe konnten wir langzeitig nicht nachweisen. Bei allen Tieren, denen auf einer Bauchseite ein Implantat mit Fibrinkleber, auf der anderen Seite ein solches ohne den Kleber eingesetzt worden war, fanden sich nach 3 Monaten keine Unterschiede im histologischen Bild. Auffallende Abstoßungsreaktionen gegen den humanen Kleber waren nicht feststellbar. 


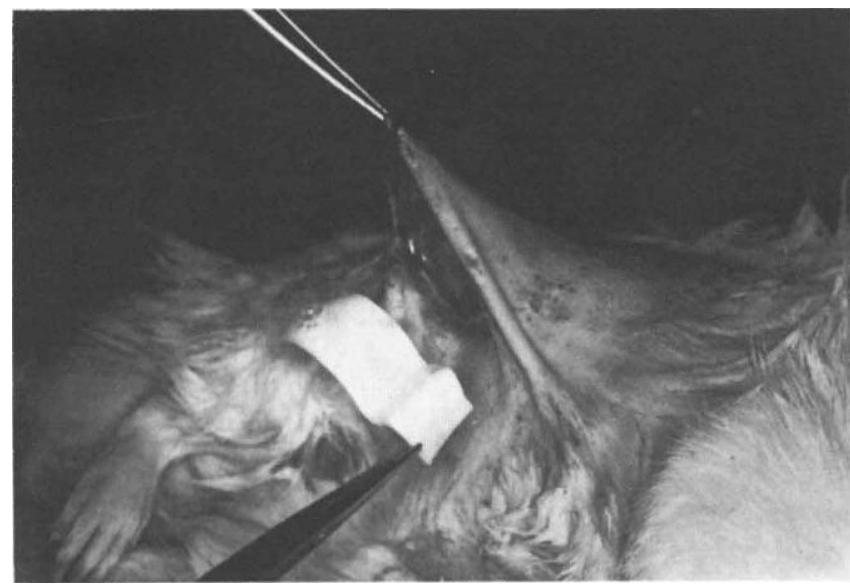

Abb. 9 Polyäthylen-Implantat, mit Fibrinkleber benetzt.

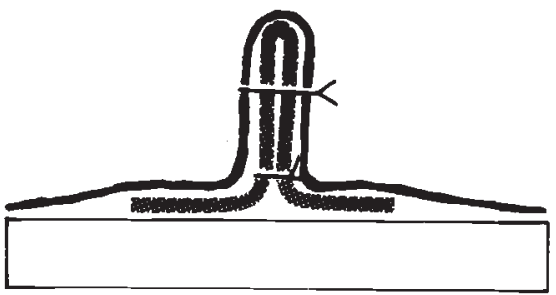

Abb. 11 Schematische Darstellung der Implantationstechnik im Hauptteil der Versuche bei den weichen Kunststoffen (Proplast, Teflon, Dacron): Die Falte im Kunststoff selbst wird durch eine Suturamidnaht gehalten, die Haut dann durch eine temporäre Matratzennaht fixiert.

Eine Faltung der nicht thermoplastischen Kunststoffe (Proplast, Teflon, Dacron) und Formgebung $\mathrm{n} u \mathrm{r}$ mit Fibrinkleber war auf Dauer nicht erfolgreich. 4 hierzu durchgeführte Implantationen, bei denen auf die Verwendung von Matratzennähten und Suturamidnähten im Kunststoff verzichtet wurde, zeigten, nachdem zunächst durch den Fibrinkleber eine verwertbare Haut-Kunststoff-Falte modelliert werden konnte, daß dieses Relief nach spätestens 3 Monaten völlig verstrichen war. Die Sektion ließ ein zwar reizlos eingeheiltes, aber flach unter der Haut liegendes Implantat erkennen.

Diese parallel zu den anderen durchgeführten Versuche bestätigten die Richtigkeit der für den Hauptteil der Untersuchung gewählten Versuchsanordnung mit Verwendung von Fibrinkleber in Kombination mit durchgreifenden $\mathrm{Ma}$ tratzennähten.

Dabei sahen wir eine makroskopisch erkennbare, eitrige Infektion in einem Fall (Teflon), Hautnekrosen mit freiliegendem Implantat 6 mal, und zwar 3 bei Teflon, 2 bei Proplast II und eine bei Polyäthylen. Diese Nekrosen traten vorwiegend im Bereich einer durchgreifenden Matratzennaht oder an der prominenten Kante der Falte auf

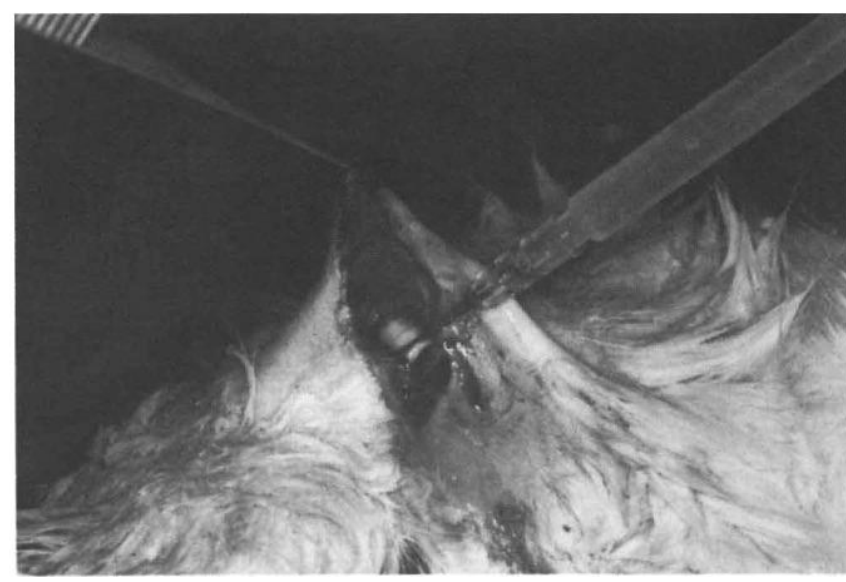

Abb. 10 Zugabe der Thrombin-Komponente zur Fibrinklebung vor Verschluß der Zugangswunde.

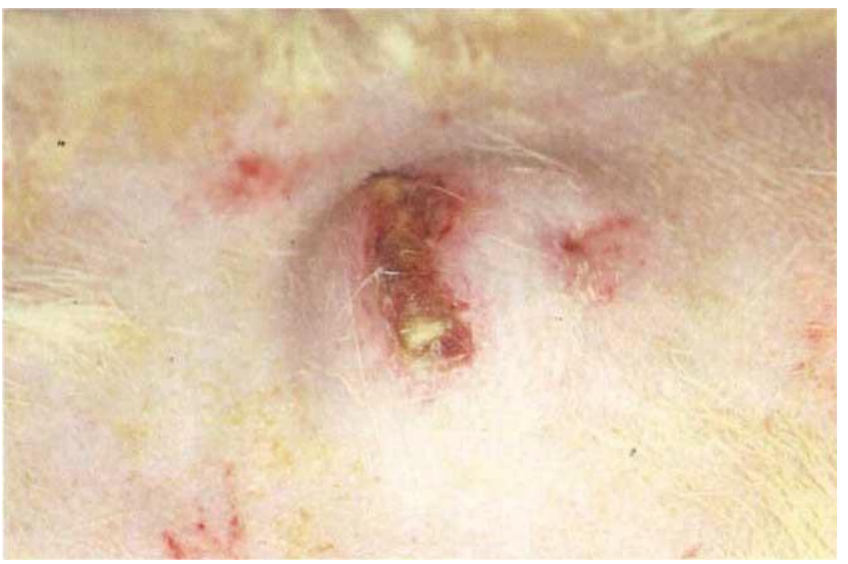

Abb. 12 Beispiel eines an der Prominenz der Falte freiliegenden Implantates (Proplast, 3 Monate nach Implantation).

(Abb. 12). Lagen sie im Bereich der Matratzennaht, so führten sie nicht zwangsläufig zur Freilegung des gesamten Implantates.

Im folgenden wird die Auswertung der Ergebnisse für die einzelnen Kunststoffe wiedergegeben:

Poröses Polyäthylen: Die Stabilität der einmal unter Erhitzen bei $150^{\circ} \mathrm{C}$ gegebenen Form war in allen Fällen sehr gut. Es konnte auch nach 8 Monaten weder makroskopisch noch histologisch eine wesentliche Änderung der Form oder des Gefüges registriert werden. Die rasterelektronenmikroskopische Untersuchung läßt vor der Implantation einerseits erkennen, daß die Poren eine Größe bis zu etwa 200 , u aufweisen können, andererseits besteht zusätzlich eine „Mikroporosität“ in der eigentlichen Kunststoffmasse mit Porenöffnungen unterhalb einer Größenordnung von etwa 4 , u (Abb. 1). Bei den letztgenannten „Mikroporen“ handelt es sich aber um geschlossene Poren.

Nach 3 Monaten war im Rastermikroskop vollständige Einbettung in Bindegewebsfasern erkennbar (Abb. 13), wobei das offene Porensystem histologisch vollkommen bindegewebig durchbaut war (Abb. 14). Einmal sahen wir eine umschriebene Hautnekrose von $0,5 \mathrm{~cm}$ Durchmesser. 


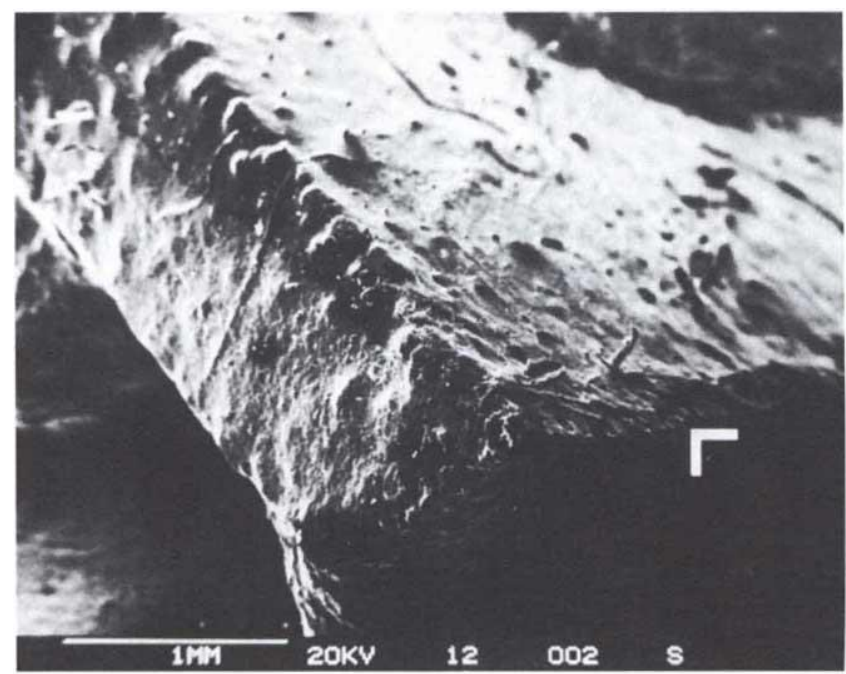

Abb. 13 In Bindegewebe eingebettetes Implantat aus porösem Polyäthylen nach 3 Monaten. An der mit Pfeil markierten Stelle Kunststoff zur Demonstration freigelegt. Rasterelektronenmikroskop; schwache Vergrößerung.

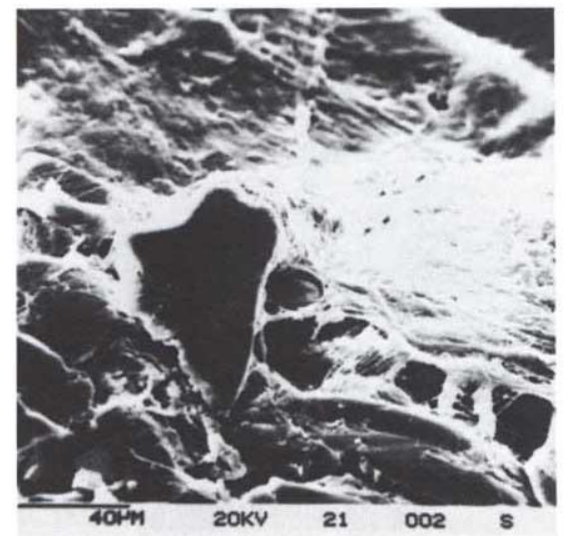

Abb. 15 Bindegewebige Einfassung von Teflon-Fasern, 3 Monate postoperativ. In der unteren Bildhälfte angeschnittener Kunststoff, Rasterelektronenmikroskop, ca. 400fach.

Der Raum unter den Wölbungen im Kunststoff war meist mit Fettgewebe ausgefüllt. Nach 10 Monaten war von 10 Implantaten eines abgestoßen.

Teflon: Hier war die Formbarkeit gut, auch die Stabilität der einmal durch Naht angebrachten Falte war zunächst ausreichend. Allerdings zeigten sich hier die meisten Nekrosen, die auch einen Durchmesser von $0,7 \mathrm{~cm}$ erreichten. Im Zusammenhang mit einer Nekrose sahen wir einmal eine eitrige Infektion. Im Rasterelektronenmikroskop ebenfalls Einbettung in ein relativ zartes Granulationsgewebe (Abb. 15). Histologisch im Vergleich $\mathrm{zu}$ den anderen Implantaten auch noch nach 10 Monaten deutlich geringere Durchbauung mit Bindegewebe, meist die zentralen Abschnitte des Kunststoffstreifens nicht mehr errei-

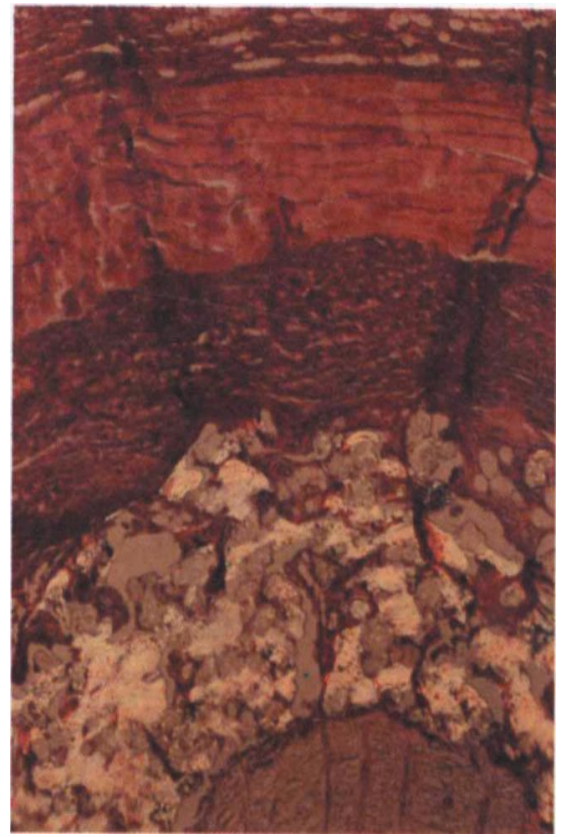

Abb. 14 Poröses Polyäthylen, 3 Monate nach Implantation. Ladewig.

chend, wo sich stattdessen gelegentlich kleine Mikroserome fanden (Abb. 16). Nach 10 Monaten waren von 11 Implantaten 2 abgestoßen, bei keinem war die gewünschte Form ausreichend gut erhalten.

Dacron: Schlechte Formstabilität und schwierige Handhabung, da es sich um ein besonders dünnes Material handelt. Das häufige Auftreten von Abflachung der Falte durch subkutanes ,Umkippen“ war auffallend. Allerdings kam es nie zu einer Hautnekrose oder anderen Heilungsstörungen. Histologisch wie bei Teflon oft keine vollständige bindegewebige Durchwachsung (Abb. 17, 18).

Proplast II: Rasterelektronenmikrospisch und histologisch liegen die Aluminiumfaserfragmente ohne feste Verbindung im Kunststoffgerüst (Abb. 19). Sie finden sich nach Monaten verteilt im einbettenden Bindegewebe, werden dort aber reizfrei eingelagert. Handhabung erschwert durch eine gewisse Brüchigkeit des Materials. Ausreichende Formstabilität, allerdings zeigten auch hier die Implantate nach spätestens 3 Monaten eine Tendenz zur Abflachung unter der Haut. Zwei Nekrosen, einmal im Bereich der Matratzennaht, einmal über der Prominenz der Falte. Histologisch bindegewebige Durchbauung nicht im gleichen Ausmaß wie bei Polyäthylen (Abb. 20, 21). Im Vergleich zu den anderen Kunststoffen auffallend viele Histiozyten und Fremdkörperriesenzellen im histologischen Bild. 3 von 10 Implantaten waren bei Abschluß der Versuche abgestoßen.

\section{Diskussion}

Die gestellten Anforderungen konnten letztlich von den textilen Kunststoffen (Dacron, Teflon, Propast II) deshalb nicht erfüllt werden, weil hier die Implantate nach Ablauf des Versuchs durch die Hautspannung abgeflacht und die Falte damit verstrichen war.

Die nach der Implantation von gefaltetem, weichem Kunststoff je nach verwendeter Technik beobachtbaren postope- 


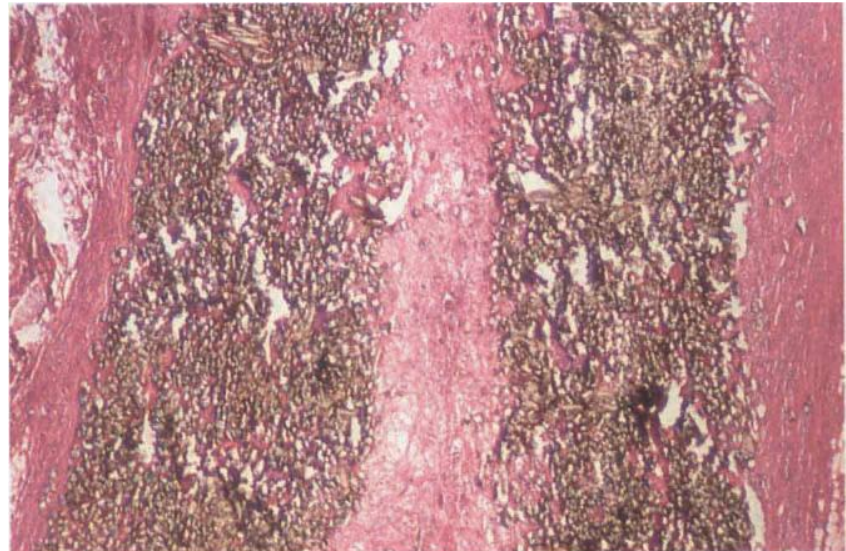

Abb. 16 Lückenhafte Durchbauung mit Granulationsgewebe bei Tefion. HE.

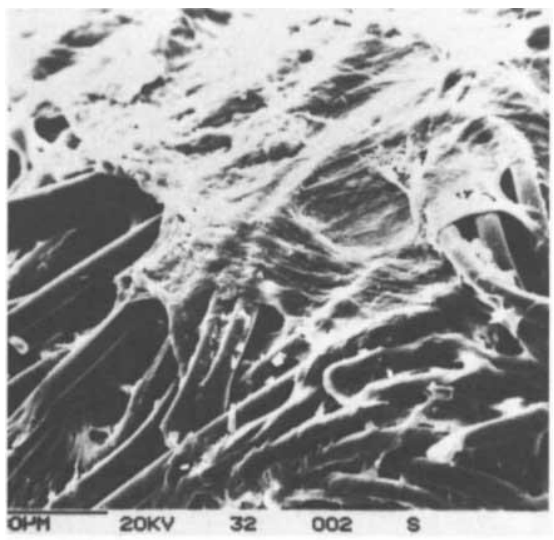

Abb. 17 Von Granulationsgewebe überzogene Dacron-Fasern, 3 Monate nach Implantation. Rasterelektronenmikroskop, ca, 200fach.

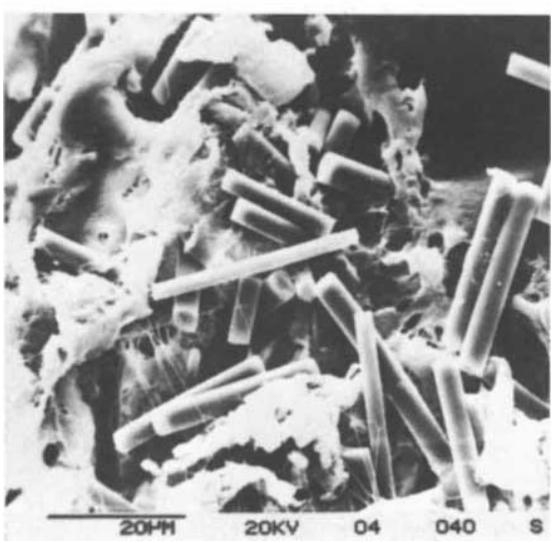

Abb. 19 Proplast II. Deutlich erkennbare Aluminiumfasern in Kunststoffgrundsubstanz. Rasterelektronenmikroskop, ca. 1000fach.

rativen Verhaltensvariationen veranschaulicht Abb. 22 (A, B, C) schematisch:

A. Formgebung und Fixierung unter der Haut unter alleiniger Verwendung von Fibrinkleber: Das Implantat entfaltet sich unter der Hautspannung und flacht völlig ab.

B. Faltung des Kunststoffs mit nicht resorbierbarem Nahtmaterial, Fixierung unter der Haut mit Fibrinkleber: Das Implantat kann sich zwar nicht entfalten, kippt aber unter der Hautspannung im Laufe von Wochen bis Monaten um. C. Faltung des Kunststoffs wie bei B, subkutane Fixierung mittels durchgreifender Matratzennaht: Die stehende Hautfalte bleibt erhalten, solange die Matratzennaht belassen wird. Nach Ziehen des Fadens jedoch gleiches Verhalten die bei $\mathrm{B}$.

Das Verstreichen der Falte durch Umkippen trat jedoch zunehmend erst nach dem 3. Monat auf (Abb. 23), weshalb die bis dahin erfolgte Beurteilung $\mathrm{zu}$ einer $\mathrm{zu}$ günstigen
Abb. 20 Bindegewebshülle bei Proplast-Implantat nach 3 Monaten. In der unteren Bildhälfte angeschnittener Kunststoff. Rasterelektronenmikroskop, ca. 50 fach. 


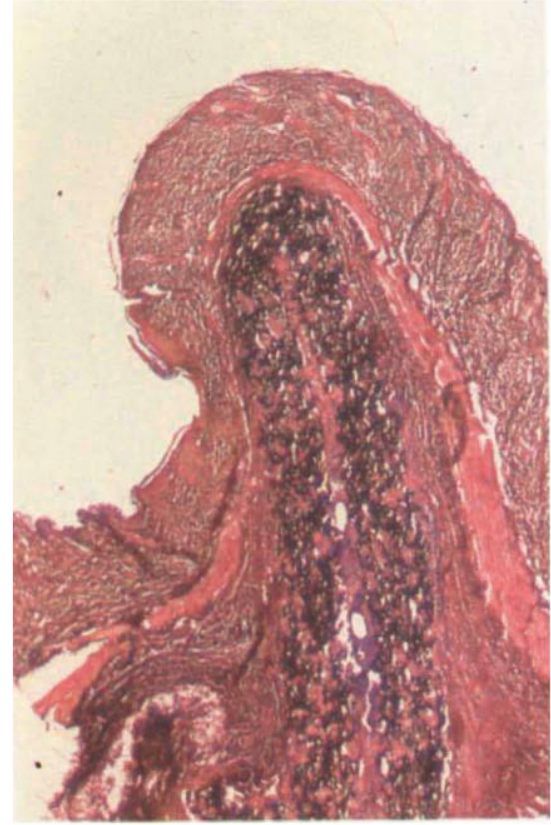

Abb. 21 Haut-Kunststoff-Falte mit Proplast II, 3 Monate nach Implantation. Ladewig.

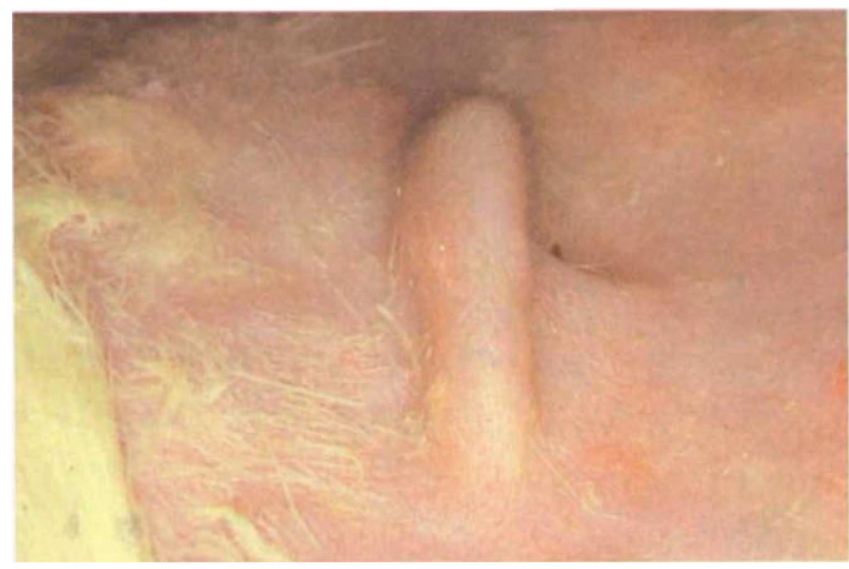

Abb. 23 Nach 3 Monaten noch gutes Ergebnis einer stehenden Hautfalte am Beispiel eines Dacron-Implantes.

Bewertung auch der weichen Kunststoffe führte (2). Besonders schnell trat das subkutane Umschlagen der Falte bei dem dünnen und weichen Dacron ein.

Die fixierenden Matratzennähte begünstigen Hautnekrosen und umschriebene Infektionen, allerdings können die histologisch erkennbaren Infektzeichen auf den unmittelbaren Bereich des Fadens beschränkt bleiben und müssen nicht zum vollständigen Verlust des Implantates führen.

Die beste Formkonstanz zeigte das thermoplastische poröse Polyäthylen, welches seine einmal bei $150^{\circ} \mathrm{C}$ gegebene Form während der Versuchsdauer völlig unverändert behielt. Dabei adaptierte sich die Haut gut an das Kunststoffrelief (Abb. 24, vgl. Abb. 8).
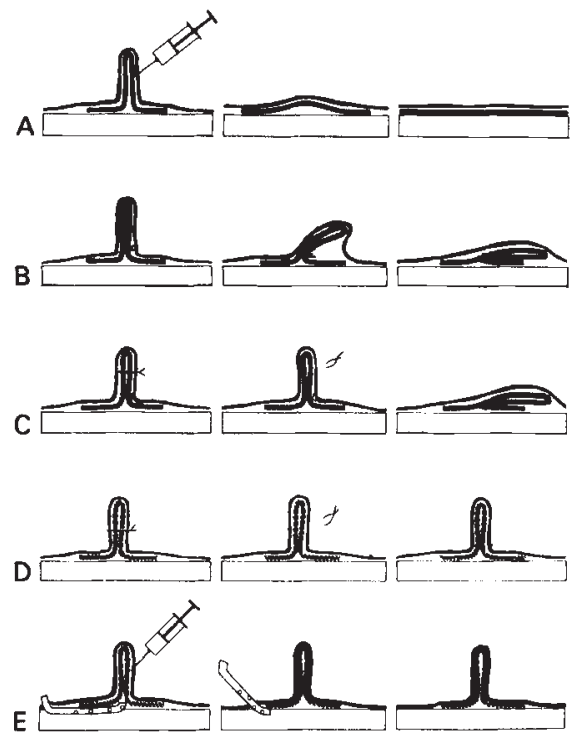

Abb. 22 Schematische Darstellung des postoperativen Verlaufes in Abhängigkeit von der Implantationstechnik. Erläuterung im Text.

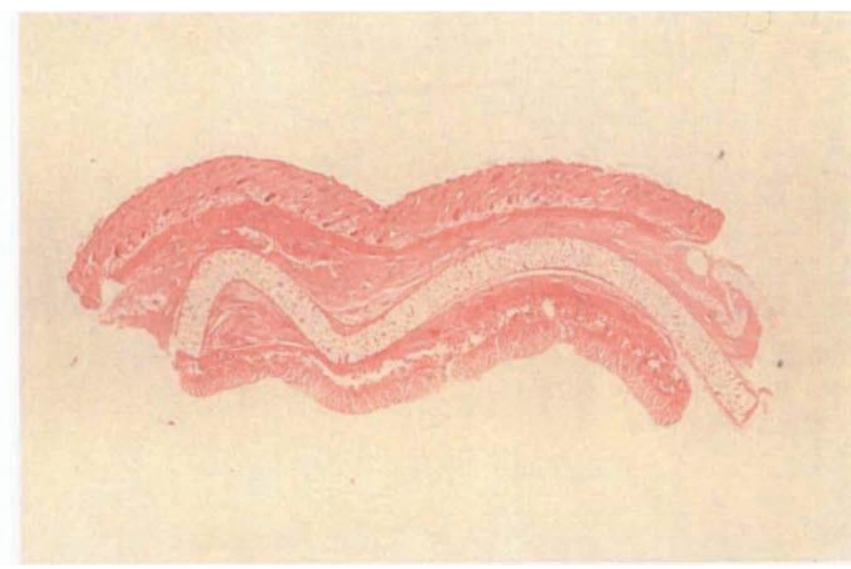

Abb. 24 Gute Formstabilität und Adaptation der Haut bei porösem Polyäthylen, 8 Monate postoperativ. HE.

Die Formstabilität des Polyäthylen macht auch das in Abb. $22 \mathrm{D}$ und $\mathrm{E}$ veranschaulichte, zu den anderen Kunststoffen unterschiedliche postoperative Verhalten der Implantate verständlich:

D. Auch nach Entfernung der Matratzennaht kann das Implantat nicht umkippen; bei guter Adaptation bleibt eine stehende Hautfalte erhalten.

E. Gleich gutes Ergebnis unter Verzicht auf die Matratzennaht bei Kombination von Fixierung mit Fibrinkleber und Saugdrainage. Diese Methode kam im klinischen Bereich zur Anwendung.

Trotz der Festigkeit dieses Materials kam es im Tierversuch nur einmal zu einer Hautnekrose. 


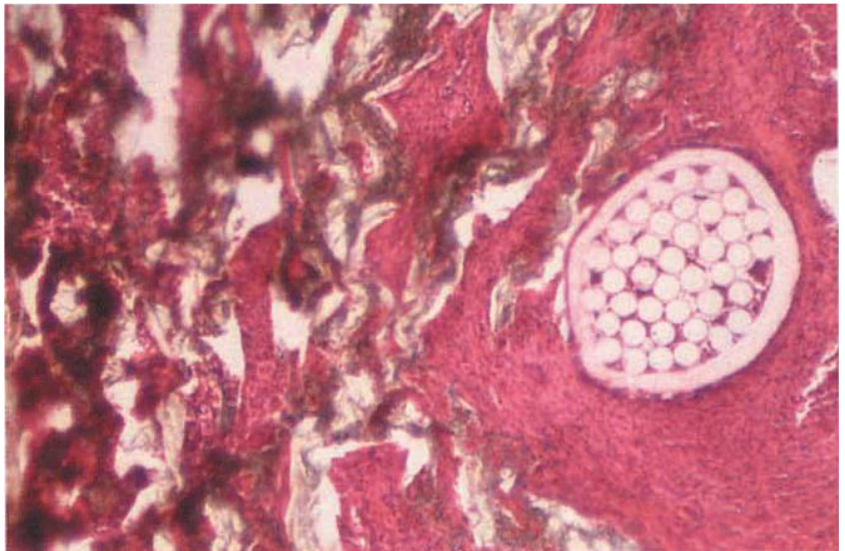

Abb. 25 Suturamidfaden neben Proplast II-Implantat. 4 Monate postoperativ. HE.

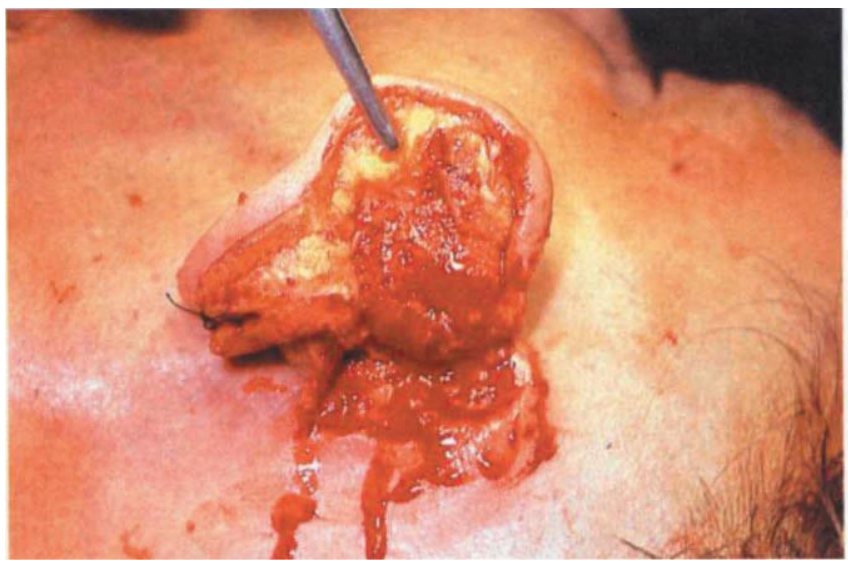

Abb. 27 Häutige Hülle der Ohrmuschel nach Resektion von nekrotisch erweichten Knorpel bei abszedierender Perichondritis. Helix nur rudimentär erhalten.

Der vor Implantation am Kunststoff selbst für die Faltung angebrachte Suturamidfaden (bei Proplast, Teflon und Dacron) führte in keinem Fall zu irgendwelchen Heilungsstörungen, was auch histologisch nachweisbar war (Abb. 25).

\section{Schlußfolgerungen}

Das von uns entworfene tierexperimentelle Modell zur Untersuchung der Eignung von Kunststoffen für die Rekonstruktion der Ohrmuschel läßt die Beurteilung der erforderlichen Kriterien zu.

Die untersuchten Kunststoffe boten zunächst prinzipiell gute Voraussetzungen für die genannte Fragestellung (Porosität, Flexibilität), bei der abschließenden Beurteilung jedoch können ausreichende Qualitäten nur bei dem thermoplastischen porösen Polyäthylen erkannt werden. Als optimale Stärke des Materials wurden im Tierversuch $1,2 \mathrm{~mm}$ ermittelt.

Das Einsetzen der Implantate in die gewünschte Position und die Adaptation der Haut werden durch die Verwen-

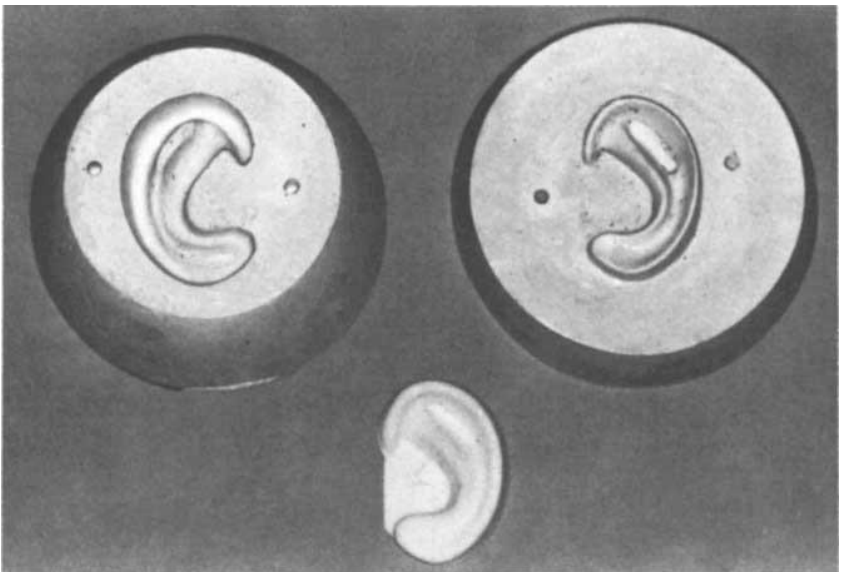

Abb. 26 Zur Herstellung einer Ohrmuschel-, Endoprothese" aus porösem Polyäthylen (unten) verwendete Formteile (oben).

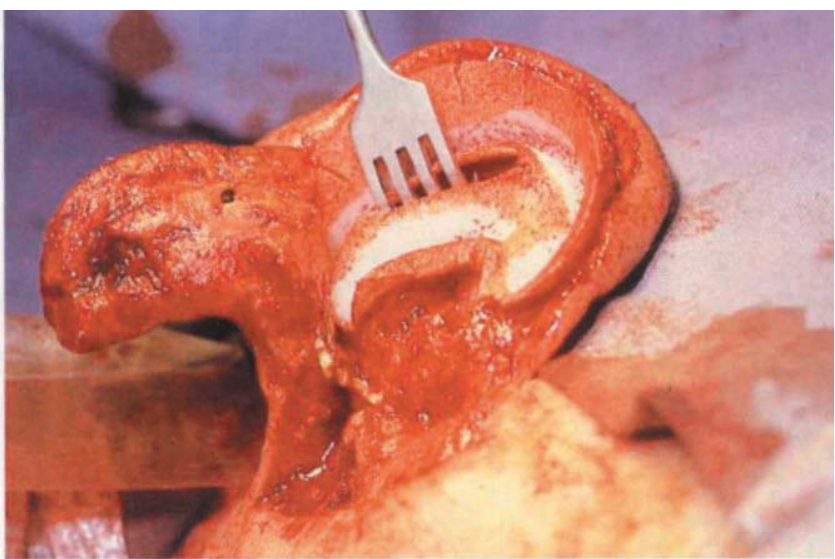

Abb. 28 Kunststoffgerüst aus porösem Polyäthylen beider Implantation.

dung von Fibrinkleber außerordentlich erleichtert. Andererseits sind für die spätere Einheilungsphase Matratzennähte oder andere mechanische Fixierungen $(z$. B. Saugdrainage nach Brent (4)) unverzichtbar. Evtl. benutzte Matratzennähte sollten so früh wie möglich entfernt werden, um Infektionen und Hautnekrosen keinen Vorschub zu leisten. Eine umschriebene Infektion muß andererseits nicht zur Entfernung des gesamten Implantates zwingen. Sollte die Entfernung jedoch notwendig werden, so ist sie bei allen hier untersuchten Kunststoffen auch nach längerer Implantationsdauer ohne Fragmentierung möglich.

\section{Klinische Anwendung}

Aufgrund der Erfahrungen im Tierexperiment $(1,2)$ haben wir für den klinischen Einsatz das poröse Polyäthylen ausgewählt. Es bietet neben der besten Formstabilität und guten Formbarkeit auch den Vorteil der leichtesten Handhabung den anderen Kunststoffen gegenüber. Die Herstellung eines Stützgerüstes für die Ohrmuschelrekonstruktion aus diesem Material ist unproblematisch (Abb. 26). Wir 


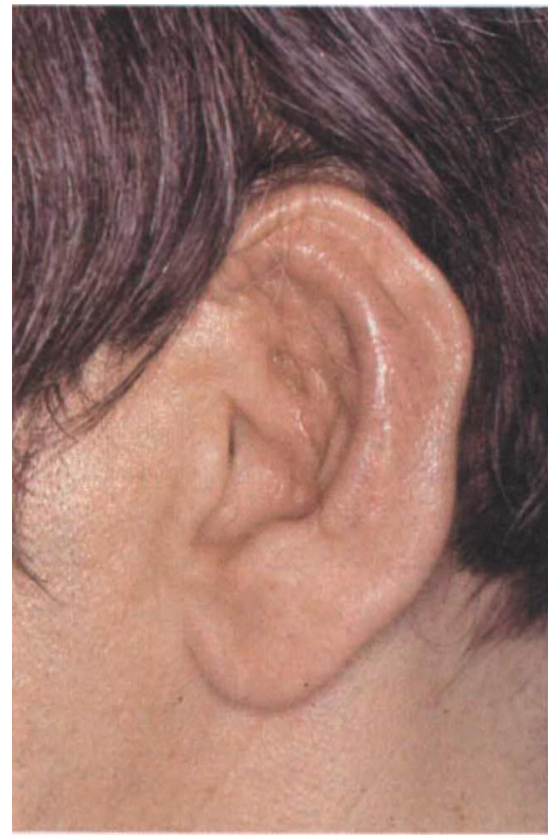

Abb. 29 Die Ohrmuschel 10 Monate postoperativ. Im Bereich der Fossa triangularis war nach 7 Monaten eine Korrektur durchgeführt worden.

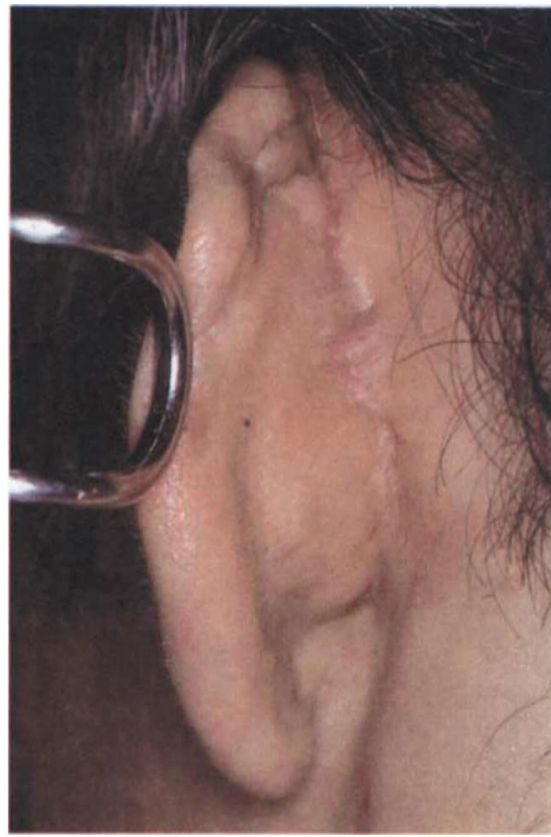

Abb. 30 Die retroaurikuläre Falte der gleichen Ohrmuschel nach 10 Monaten.

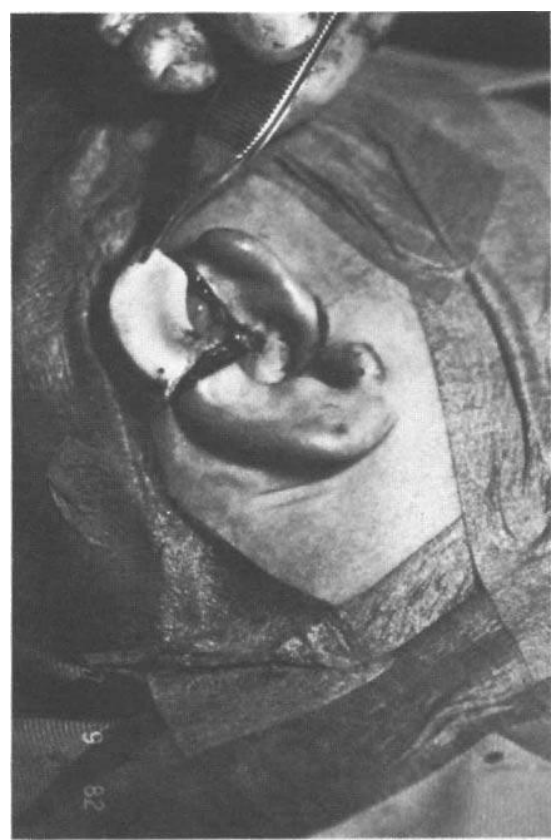

Abb. 31 Zur Korrektur einer Mikrotie verwendetes, geformtes Fragment aus porösem Polyäthylen. * konnten u.a. eine durch abszedierende Perichondritis nahezu verlorene Ohrmuschel durch Einsetzen einer solchen „Endoprothese“ rekonstruieren. In dem betreffenden Fall war nach Ausräumung von nekrotisch zerfallenden Knorpelmassen nur die häutige Hülle der Ohrmuschel erhalten geblieben (Abb. 27). In diese Hülle wurde das thermoplastisch geformte Kunststoffgerüst implantiert (Abb. 28). In der postoperativen Phase zeigte sich zwar, daß durch einen $Z$ weiteingriff das Implantat verkleinert werden mußte - es traten Nahtdehiszenzen auf - im übrigen aber war die Einheilung ungestört. Das kosmetische Ergebnis wurde nach 7 Monaten durch Korrektur eines unphysiologischen Balkens im mittleren Drittel der neuen Ohrmuschel nochmals verbessert (Abb. 29, 30).

Auch im Fall einer Mikrotie wurde ein Implantat aus porösem Polyäthylen bei einem 12 jährigen Mädchen verwendet. Das von R. Meyer in Lausanne zusammen mit dem Autor operierte Ohr (Abb. 31) zeigte ein halbes Jahr nach dem Aufbau gute Einheilung des Kunststoffs ohne Dislokation oder Hautschädigung.

* Der Autor dankt Herrn Priv. Doz. Dr. Rudolph Meyer, Lausanne, für die freundliche Überlassung der Aufnahme.

\section{Literatur}

(1) Berghaus, A., M. Handrock: Fibrinvernetzte Knorpel- und Kunst- stoffspäne zur Ohrmuschelrekonstrukrion. Arch Otorhinolaryngol. 231 (1981) 601-605
(2) Berghaus, A., M. Axhausen, M. Handrock: Zur Verwendbarkeit von Kunststoffen bei der Rekonstruktion der Obrmuschel. Arch. Otorhinolaryngol. 235 (1982) 622-625

(3) Breadon, G. E., E. B. Kern, H. B. Neel: Autografts of crushed and uncrushed bone and cartilage. Arch. Otolaryngol. 105 (1979) 75-80

(4) Brent, B.: The correction of Microtia with Autogenous Cartilage Grafts: I. The Classic-Deformity. Plast. Reconstr. Surg. 66, 1 (1980) 1-12 (5) Brown, B. L., E. B. Kern, H. B. Neel: Transplantation of fresh allografts (homografts) of crushed and uncrushed cartilage and bone: a 1-Year analysis in rabbits. Laryngoscope 90 , 9 (1980) 1521-1533

(6) Cronin, Thomas D.: Use of a Silastic frame for Construction of the Auricle. In: Tanzer, R. C., Edgerton, M.

T. Symposium on reconstruction of the auricle. Mosby, Saint Louis (1974) 33-45

(7) Fukuda, O., Y Atsushi: Reconstruction of the Microtic Ear with Autogenous Cartilage. Clin. Plast. Surg. 5, 3 (1978) 351-366

(8) Gorney, M., S. Murphy, E. Falces:
Spliced autogenous conchal cartilage in secondary ear reconstruction. Plast. Reconstr. Surg. 47, 5 (1971) 433-437

(9) Matthews, D. N.: Reconstruction of the Ear. Fortschr. Kief. Ges. Chir. 7 (1961) 96-101

(10) Meyer, R.: Über Ersatzplastik der Ohrmuschel. Fortschr. Kief. Ges. Chir. 2 (1956) 176-179

(11) Meyer, R.: Persönliche Mitteilung, Lausanne 1981

(12) Ohmori, S.: Reconstruction of the microtic ear by use of a silicone rubber frame. Plast. Reconstr. Surg. 56 (1975) 352

(13) Peer, L. A.: Reconstruction of the auricle with diced cartilage grafts in a vitallium ear mold. Plast. Reconstr. Surg. 5 (1948) 653-666

(14) Spina, V., L. Kanakura, J. M. Psillakis: Total reconstruction of the ear in congenital microtia. Plast. Reconstr. Surg. 48 (1971) 349-357

(15) Tanzer, R. C.: Microtia. Clin. Plast. Surg. 5 (1978) 317-336 (16) Weerda, H.: Unsere Erfahrungen mit der Chirurgie der Obrmuschelmißbildungen. Laryngologie, Rhinologie, Otologie 9 (1982) 493-500

\section{Dr. Alexander Berghaus}

Klinikum Steglitz der FU Berlin Hals-Nasen-Obren-Klinik und Poliklinik Hindenburgdamm 30 1000 Berlin 45 